J3eA, Journal sur l'enseignement des sciences et technologies de l'information et des systèmes,

Volume 3, Hors-Série 1, 10 (2004)

DOI : http://dx.doi.org/10.1051/bib-j3ea:2004610

(C) EDP Sciences, 2004

Dimensions technique et psychologique d'une assistance robotisée pour la compensation des incapacités physiques

L. Delahoche ${ }^{1}$, O. Rémy-Néris ${ }^{2}$, J.M. Devauchelle ${ }^{1}$, D. Nadalini ${ }^{2}$, B. Marhic ${ }^{1}$ et É. Brassart ${ }^{1}$

${ }^{1}$ Université de Picardie Jules Verne

IUT Amiens

Départements Génie Mécanique et Productique et Informatique avenue des facultés

F-80025 Amiens CEDEX 1, France

${ }^{2}$ Groupe Hopale, Centre Calvé

72 esplanade Parmentier

F-62608 Berck-sur-Mer, France 


\title{
Dimensions technique et psychologique d'une assistance robotisée pour la compensation des incapacités physiques
}

\author{
Laurent Delahoche , Olivier Rémy-Néris $\uparrow$, Jean Marc Devauchelle \\ Dominique Nadalini $\uparrow$, Bruno Marhic Eric Brassart \\ Aniversité de Picardie Jules Verne \\ IUT Amiens, Départements Génie Mécanique et Productique et Informatique \\ Avenue des facultés, 80025 AMIENS Cedex 1 \\ $\checkmark$ Groupe Hopale - Centre Calvé \\ 72 esplanade Parmentier, 62608 BERCK sur MER
}

\begin{abstract}
Résumé - Cet article présente un projet financé par la région Picardie qui a pour objectif d'intégrer de nouvelles fonctionnalités pour l'assistance aux personnes à mobilité réduite. Il consiste à étudier l'impact technique, psychologique et clinique d'une approche originale qui consiste à embarquer un bras préhenseur sur une base mobile. La spécificité de notre approche réside la stratégie maître-esclave déployé pour déplacer cet ensemble mobile par rapport au fauteuil du patient. Le bras préhenseur utilisé dans cette première version est bras MANUS.
\end{abstract}

Mots clés : assistance, déficience, psychologie, robotique, préhension, navigation.

\section{INTRODUCTION}

La robotique d'aide aux personnes handicapées est apparue en France en 1984 avec le bras manipulateur Spartacus, puis en 1985 avec le programme MASTER (Manipulateur Autonome au Service des Tétraplégiques pour l'Environnement et la Réadaptation). Le robot MANUS® a été commercialisé en 1985 par la société Exact Dynamics en Hollande grâce à une commande du gouvernement hollandais. Sa diffusion a commencé en France grâce à l'association française contre la myopathie dès 1988 pour de premiers essais. Aux Etats unis le programme DEVAR a été développé par l'université de Standford. Il n'a jamais aboutit à une commercialisation en particulier en raison du coût du robot utilisé (robot Puma $\left.{ }^{\circledR}\right)$. En Angleterre, le robot Handy est commercialisé. Il totalise le plus grand nombre d'unités vendues au monde. Il est dévolu à la prise de repas avec des tâches préprogrammées.

L'association APPROCHE (Association pour la promotion de la robotique concernant les personnes handicapées) a évalué en 1996 et 1997 la station robotique RAID MASTER II et le robot Manus II sur une centaine de personnes handicapées en France. Toutefois peu de robots (moins de 10) ont été commercialisés ou mis à disposition des personnes handicapées sur le territoire français.

Des laboratoires de recherche en robotique, tels le LASC (Laboratoire d'Automatique des Systèmes Coopératifs) de Nancy, ainsi que des instituts médicaux comme le centre de Garche ou le groupe Hopale, travaillent depuis de nombreuses années sur le problème $\mathrm{du}$ handicap, et sont reconnus nationalement sur ce créneau. Des associations telles que l'AFM (l'Association Française contre les Myopathies), l'APF (association des paralysés de France), des groupes de recherche comme 1'IFRATH (Institut Fédératif de Recherche sur les Aides Techniques pour Handicapes) se sont créées et drainent une réelle dynamique tant d'un point de vue humain que technique au niveau de la supervision de projets ambitieux dans le domaine de l'assistance technique. Ainsi des projets nationaux tels que ARPH (Assistance à la personne handicapée par un système robotique semiautonome) [1] ou MASTER [2] montrent un réel intérêt et une volonté certaine pour faire progresser la problématique de l'assistance technique pour les personnes à mobilité réduite. On peut identifier globalement trois types d'approches robotisées liées à l'assistance technique pour personnes handicapées: les bras préhenseur à base fixe, les fauteuils roulants embarquant un bras préhenseur et enfin les fauteuils roulants intégrant des fonctionnalités d'aide au déplacement.

Dans le cadre de la première approche, le projet MASTER mené par le C.E.A. (Commissariat à l'Energie Atomique) met en œuvre une station robotisée à poste fixe pilotée par une interface de commande unique adaptée au handicap de l'opérateur (tétraplégique fonctionnel) [2]. En effet, la mise à disposition, auprès de personnes à mobilité réduite, de systèmes d'aide robotisés, particulièrement sophistiqués, représente une avancée importante qui est censée se traduire par une amélioration de la vie quotidienne de ces personnes. Associés à d'autres aides, ces systèmes, qui ne se substituent pas à l'aide d'une tierce personne, ont pour objectif de faciliter l'autonomie de la personne déficitaire en la libérant de certaines contraintes et attentes.

Cependant, la maîtrise de ces aides technologiques pose un certain nombre de problèmes qui sont de l'ordre de l'ergonomie cognitive dans le mesure où ils concernent précisément l'attitude spécifique de la personne handicapée dans sa relation avec la machine (robot) et l'environnement dans lequel le binôme homme-machine évolue. Ces problèmes cumulent la dimension technique (liée aux possibilités du robot et à ses 


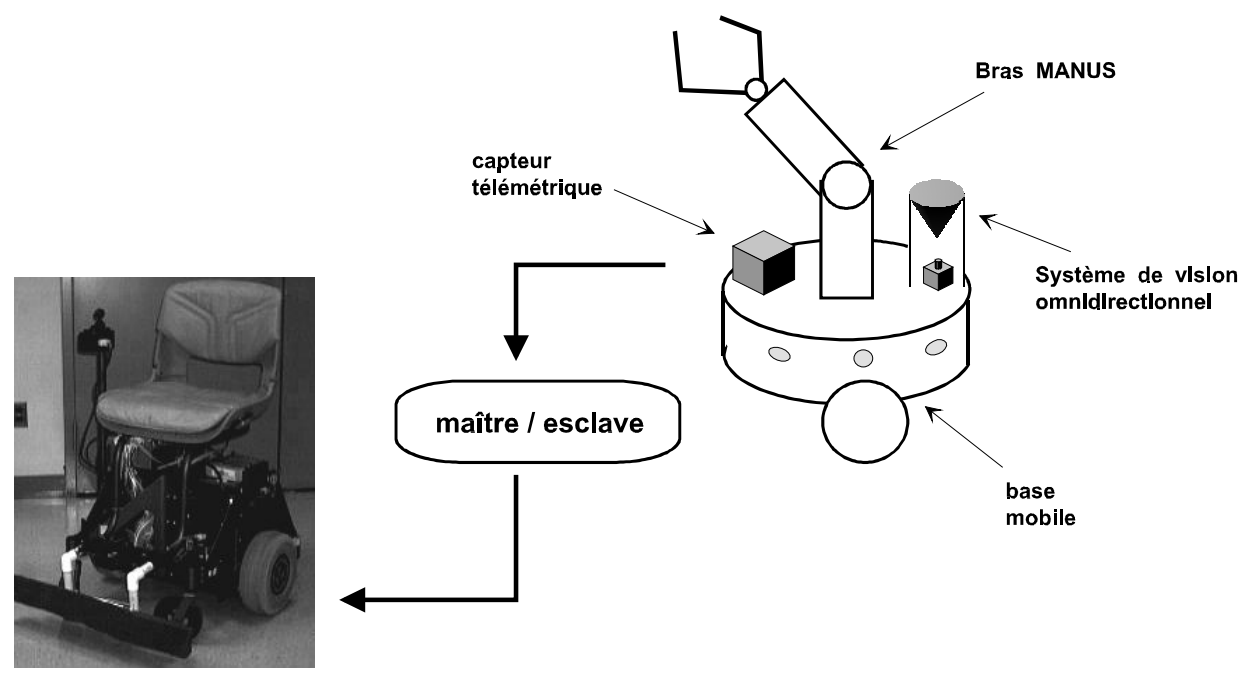

Fig.1. L'assistance robotisée.

contraintes d'utilisation) et la dimension humaine (liée aux représentations de cette prothèse (système robotisé) et à l'impact de ces représentations sur les mécanismes d'apprentissage relatifs au fonctionnement d'un tel système d'aide).

Le point d'entrée de notre étude est une réponse à une demande sociétale concernant l'assistance technique pour le handicap en nous appuyant sur le constat mis en évidence précédemment. La nature hautement pluridisciplinaire de celle-ci a pu être valorisée dans le cadre d'un appel à projet régional HTSC (Homme Technologies et Systèmes Complexes) visant à faire coopérer les disciplines SHS (sciences humaines et sociales) et SPI (sciences pour l'ingénieur) par rapport à des problématiques entrant dans un des deux axes mobilisateurs de l'UPJV (Université de Picardie Jules Verne), il s'agit de l'axe HTSC. Les conditions d'éligibilité de cet appel étaient essentiellement liées aux deux contraintes que sont: la réponse à une demande sociétale et l'implication forte de partenaires socioéconomiques.

\section{CONTEXTE SCIENTIFIQUE DU PROJET}

L'étude que nous menons se propose de mettre en œuvre une assistance robotisée de type «maître/esclave » et d'en évaluer l'impact psychologique mais aussi et surtout l'apport clinique sur différentes populations de personnes déficientes. $\mathrm{Ce}$ projet fortement pluridisciplinaire est évidemment connexe au vaste champ qu'est celui des aides techniques pour le handicap. En outre, il est complémentaire du projet ARPH (Assistance à la personne handicapée par un système robotique semiautonome) [1] sur lequel travaille le LSC - CEMIF depuis plusieurs années.

D'un point de vue technique, l'assistance consistera à coupler une plate-forme mobile à un bras préhenseur utilisé classiquement en robotique pour handicapés. Dans un premier temps nous utiliserons le bras robot MANUS mais l'étude sera généralisable à d'autres types de bras préhenseur. L'association de ces deux technologies a pour ambition d'étendre les possibilités d'assistance, possibilités jusqu'ici restreintes à un rayon d'action limité et situé autour de la base fixe du bras robot. L'aide robotisée qui sera développée est basée sur une approche novatrice qui est une stratégie maître/esclave entre le fauteuil roulant et l'assistance technique. Cette stratégie originale devra permettre d'intégrer les deux fonctionnalités suivantes :

- le suivi automatique du fauteuil par la base mobile transportant le bras (figure 1)

- le dégagement automatique de la base mobile et du bras lorsque le patient souhaite se servir de l'assistance technique pour effectuer de la préhension d'objets en mode téléopéré (figure 1).

L'aspect commande en mode téléopéré, qui n'est pas directement lié à la stratégie « maître / esclave », constitue un troisième point important qui sera pris en compte dans notre étude par rapport au système de perception embarqué. Le projet ARPH aborde ce point de façon très spécifique et approfondie.

Les deux aspects réunis, à savoir une stratégie maître/esclave et une assistance robotisée indépendante du fauteuil, semble être un concept intéressant et novateur puisqu'il n'existe pas à l'heure actuelle de tel système sur le marché du matériel médical. Ce couplage offre de multiples perspectives et pose de nombreuses problématiques tant d'un point de vue technologique que psychologique, neuropsychologique et bien sûr clinique. Les nouvelles perspectives offertes par cette approche sont :

D'un point de vue fonctionnel, un accroissement évident du domaine de préhension,

D'un point de vue psychologique, il est probable que le bras robot puisse être perçu comme moins pesant et plus utile, ce qui peut représenter un facteur d'acceptation important. En outre, elle offre un champ d'investigation notamment lié au prolongement d'un membre à des espaces non traditionnellement appréhendés par le patient. 
Ces deux aspects montrent clairement l'importance d'articuler le projet autour de trois dimensions interdépendantes :

Technologique, avec la connaissance des solutions techniques applicables dans le cadre d'un tel projet

Psychologique et cognitive, avec la connaissance du fonctionnement psychologique de la personne déficiente eu égard aux aspects neuropsychologiques et développementaux de son fonctionnement cognitif.

Médicale et clinique, avec la connaissance pratique du problème du handicap

Les fonctionnalités et l'interface de commande existantes du bras préhenseur MANUS seront reprises intégralement.

\section{MISE EN EUVRE TECHNOLOGIQUE (SPI)}

Dans cette étude, la partie SPI se propose d'apporter ses compétences en matière de robotique mobile et de perception de l'environnement. Si le couplage d'un bras préhenseur sur une base mobile a déjà été étudié pour des applications très spécifiques liées au domaine industriel [3], sa mise en œuvre par rapport au domaine qu'est la robotique pour handicapés prend une toute autre dimension que la simple association d'un bras et d'une base mobile. Nous le disions précédemment, la dimension humaine étant ici omniprésente et prépondérante, la sophistication des choix technologiques se trouve limitée et fortement contrainte par le problème de l'interaction homme-machine.

A) - La première partie de l'étude consiste donc à choisir un système de perception le plus pertinent et surtout le plus robuste possible. La pertinence de la solution d'intégration choisie sera fonction d'une part de l'étude menée parallèlement par les neuropsychologues, psychologues et ergothérapeutes et d'autre part fonction de la stratégie maître-esclave déployée. Nous avons à ce jour décidé de nous appuyer sur une solution de perception basée sur l'utilisation de plusieurs capteurs de vision omnidirectionnelle [6]. Cette intégration ne peut évidemment pas être dissociée de la problématique qu'est la fusion de données. Cet aspect fusion induira une robustesse du système de navigation qui est directement liée à la sécurité de déplacement de la base mobile en mode autonome.

B) - La seconde partie concerne le suivi de cibles. Il est lié à la stratégie maître/esclave précédemment exposée. Le problème sera ici de faire suivre le fauteuil par la base mobile lorsque le patient se déplace et qu'il n'a pas besoin du bras préhenseur. Les approches développées dans les travaux de thèse [4][5] sur l'association de données télémétriques et visuelles omnidirectionnelles semblent très intéressantes pour résoudre la problématique du suivi. La localisation relative par rapport au fauteuil et l'évitement d'obstacles lors du déplacement seront les deux fonctionnalités prioritaires à intégrer.
C) - La troisième partie de l'étude portera sur une stratégie de dégagement par rapport au fauteuil roulant pour se mettre à disposition du patient. Cette problématique fera typiquement appel aux précédents modules. La base mobile contournera le fauteuil par rapport à l'espace libre. La mise à disposition aura pour objectif final de réaliser des missions de préhension à distance. Nous pouvons préciser à ce niveau que ces missions seront en mode totalement télé-opéré, aussi bien pour la préhension que pour le déplacement de la base.

\section{MISE EN CEUVRE PSYCHOLOGIQUE (SHS)}

En ce qui concerne la démarche psychologique d'évaluation clinique de l'appropriation de systèmes d'aide automatisés par une population de personnes à mobilité réduite, la revue de question, à un niveau théorique, nous a permis d'étayer notre interrogation relative à l'adaptabilité de la machine à son utilisateur potentiel qu'est la personne à mobilité réduite. Dans ce cadre, nous sommes en mesure de dégager trois points fondamentaux.

A) - Dans un premier temps, il nous apparaît primordial d'identifier les attentes de cette population particulière et de confronter ces attentes à la réalité des systèmes automatisés d'aide déjà existants.

B) - En second lieu, il apparaît nécessaire d'effectuer une évaluation psychologique de cette population, en testant les fonctions cognitives supérieures : Praxies, planification, fonctions exécutives, mémorisation, perception de l'espace distal et/ou proximal. En effet, l'intégrité de ces différentes fonctions n'est bien souvent pas estimée par défaut d'outils accessibles aux personnes à mobilité réduite (voire tétraplégiques).

C) - Enfin, nous considérons que la faible appropriation de la machine, par les personnes handicapées, est en partie liée à la difficulté d'apprentissage de sa manipulation. Ainsi, afin d'optimiser cette appropriation il semble important de préciser les modalités d'apprentissage des personnes à mobilité réduite afin de construire un «modèle de l'apprenant » permettant d'adapter l'interface hommemachine en simplifiant les modalités de manipulation.

Pour mettre en œuvre cette démarche il convient de procéder de différentes manières selon les objectifs fixés. En ce qui concerne le premier objectif, nous procéderons sur la base d'un questionnaire et d'une démarche d'interrogation clinique des personnes à des moments différents de leurs cursus thérapeutiques, et ce, afin d'identifier les représentations qu'elles se font de l'aide technologique. Pour ce qui concerne le second objectif, la procédure expérimentale est différente et repose sur l'utilisation d'échelles psychométriques classiques. En ce 

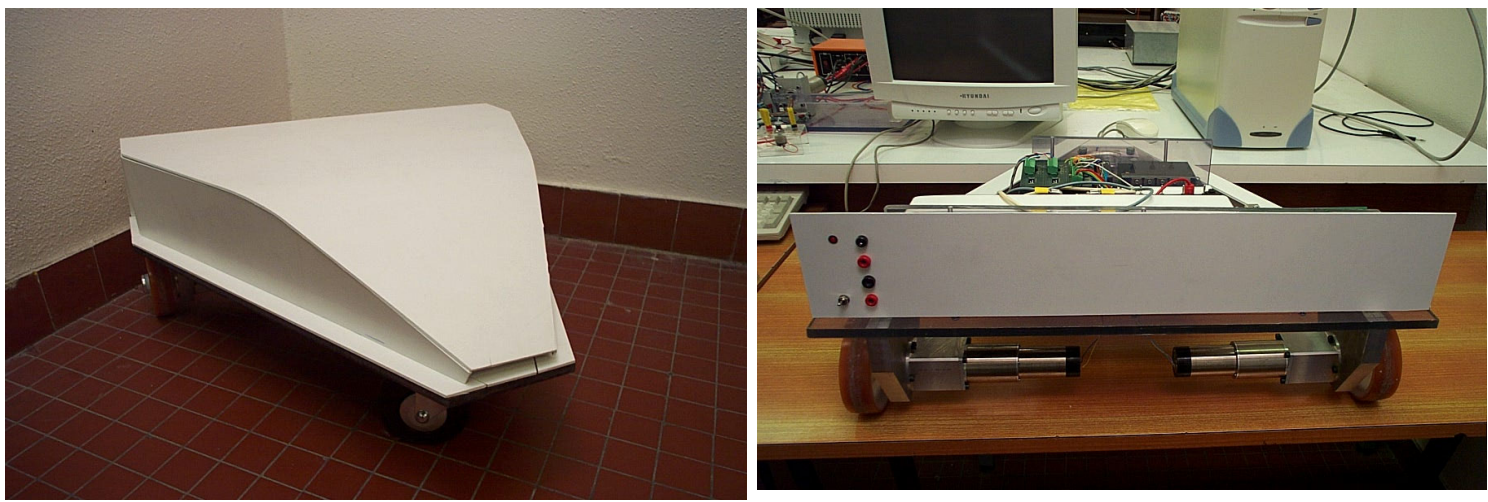

Fig.2. Prototype du porteur.

qui concerne le troisième objectif, la démarche sera encore différente et repose sur l'analyse de la tâche à laquelle est confronté l'apprenant. La finalité de cette analyse est de construire un "modèle de l'apprenant » permettant d'adapter l'interface homme-machine en simplifiant les modalités de manipulation.

\section{PARTIE CLINIQUE ET MEDICALE}

Enfin, dans notre étude, la validation clinique de l'aide robotisée développée sera prise en charge par le Centre Jacques Calvé du groupe Hopale, situé à Bercksur-Mer. Nous pouvons rappeler à ce niveau que le centre Calvé constitue le référant de ce projet puisqu'à l'origine de l'idée novatrice de couplage base mobile/bras et possèdant les connaissances cliniques sur le segment de la rééducation et de l'assistance aux personnes lourdement handicapées.

L'objectif principal du groupe Hopale dans cette étude (centre Jacques Calvé, Fédération des blessés médullaires) est de permettre à des personnes présentant un très haut niveau de dépendance physique (non usage des membres supérieurs et inférieurs) d'utiliser les moyens technologiques disponibles pour une indépendance la plus complète possible. Les progrès des fauteuils roulants électriques tant en ce qui concerne la base mobile que l'interface de commande, ont été considérables ces dernières années. Cette évolution permet actuellement de proposer une solution technique de type fauteuil roulant électrique à toute personne présentant les capacités cognitives d'assurer la fonction de déplacement du corps dans l'espace.

La mise à disposition de bras robotisés fixes ou installés sur le fauteuil roulant électrique, dans les dix plus grands centres de rééducation français pour personnes tétraplégiques n'a pourtant été suivie que d'un nombre extrêmement faible de demandes de financement pour de tels outils. Bien que les performances techniques de ces outils sont élevées, les causes psychologiques expliquent certainement en partie de cette carence d'utilisation [7]. Toutefois, d'autres causes peuvent facilement être identifiées dans notre expérience. L'une d'entre elle est l'encombrement supplémentaire des bras robotisés placés sur le fauteuil roulant électrique.

Ces différents points pourraient être résolus par un robot mobile, esclave du fauteuil roulant électrique et utilisable à volonté par le sujet handicapé dans son champ de vision.

Cette partie du projet se décompose donc en trois points :

A) - Aider à la détermination des éléments techniques pertinents en particulier pour l'interface utilisateur et les fonctionnalités de la base mobile. Cette partie se reporte à la partie technologique du projet. La connaissance du comportement des utilisateurs de fauteuil pourra permettre d'orienter certains choix pour déterminer le comportement de la base mobile porteuse du bras manus.

B) - Evaluer l'efficience de la base mobile sous forme de prototype auprès de personnes handicapées accueillies au sein du groupe Hopale. Une évaluation auprès des personnes handicapées après une phase de test par des sujets normaux installés dans des fauteuils roulants électriques sera nécessaire [8].

C) - Evaluer l'impact de la dé-solidarisation du Manus et du fauteuil roulant sur le niveau d'utilisation et l'acceptabilité des patients. L'impact de la configuration robot sur une base mobile sur le niveau d'acceptabilité du système par des personnes n'ayant jamais utilisé le robot dans la configuration initiale sera évalué sur une population de sujets tétraplégiques pouvant théoriquement bénéficier d'un robot.

\section{RESULTATS EXPERIMENTAUX}

Les avancés du projet portent principalement sur le choix d'une solution de perception à embarquer, le développement d'une première version de base mobile et enfin sur le développement d'application d'aide à l'évaluation neuropsychologique des patients concernés par l'assistance.

La partie " conception et réalisation », supervisé par Jean Marc Devauchelle, s'est chargé de l'élaboration mécanique, électrotechnique et électronique du porteur 

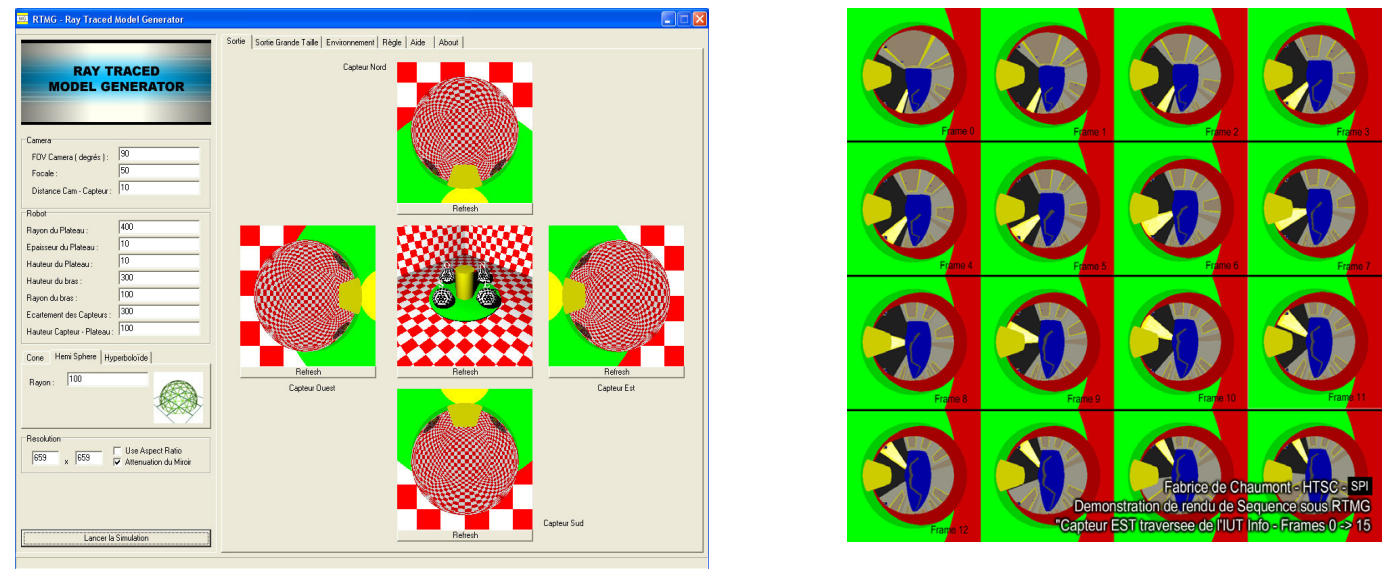

Fig.3. Séquences d'images obtenues au cours d'un déplacement.

embarquant le bras MANUS. Ils ont à ce jour développé un prototype de base mobile permettant d'embarquer une charge relativement conséquente. La solution élaborée est sous-tendue par une expérience importante acquise par l'équipe " conception » du département génie mécanique et productique sur de nombreux contrats industriels portant sur la «mécatronique». Le prototype a été conçu et réalisé par rapport aux deux paramètres qui ont été étiquetés comme prépondérant lors de la première réunion plénière :

․ le coût de la solution technique

a l'utilisation la plus poussée possible de composants standards et intégrés

La partie effecteur est basée sur l'utilisation de moteurs à courant continu et une cinématique de type «char» : les roues sont motrices et directrices. La partie puissance est entièrement conçu autour de composants intégrés qui assurent la partie commande.

La base étant développée, les algorithmes de navigation en boucle ouverte aussi, le choix de la solution de perception se posait: la stratégie de perception optimale au sens des deux critères précédemment exposés semble reposée sur l'utilisation de plusieurs systèmes de vision omnidirectionnels. La configuration embarquée et le paramétrage géométrique se devait de faire l'objet d'une simulation la plus poussée possible. L'acquisition des capteurs ne saurait en effet souffrir d'une quelconque erreur qui a posteriori ne nous permettrait pas d'atteindre les objectifs visés. Notre doctorant s'est donc attaché à développer une simulation dynamique $3 \mathrm{D}$ permettant à l'assistance robotisée de se déplacer dans un environnement intérieur modélisé correspondant à un contexte expérimental réel. Le paramétrage nous a permis de configurer :

- la géométrie du ou des capteurs,

- le nombre de capteurs à embarquer,

- le positionnement du ou des capteurs sur la base mobile.

Le simulateur nous a permis d'entériner une solution fiable basée sur l'utilisation de trois capteurs omnidirectionnels placés autour du bras préhenseur. Il est à noter que nous obtenons des séquences sensorielles de synthèse correspondant à ce qui est observé par chacun des capteurs.

\section{CONCLUSION}

La forte cohésion du groupe de travail du projet est garante de la pérennité du projet à long terme. En outre il est important de noter après ces quelques mois de passés sur ce projet, que les compétences complémentaires des acteurs ont été clairement mises en valeur. Cette complémentarité permettra indubitablement une prise en compte globale du problème du handicap qui devrait aboutir à une solution d'assistance la plus optimale possible.

La mise en œuvre du prototype de la base mobile montre la capacité des équipes de travail à lever de nombreux verrous technologiques inhérents à toute réalisation. Les travaux à venir porteront surtout sur l'utilisation de la stratégie de perception pour permettre à la base mobile de suivre le fauteuil du patient. Le travail d'évaluation psychologique de la population considérée est en cours. Le dépouillement des résultats en sera la prochaine étape.

\section{REFERENCES BIBLIOGRAPHIQUES}

[1] P. Hoppenot, E. Colle : « Real-time localisation of a low-cost mobile robot with poor ultrasonic data »Control Engineering practice, IFAC journal, 1998, pp 925-934.

[2] M. Busnel, R. Cammoun, F. Coulon-Lauture, J.M. Détriché, G. Le Claire, B. Lesigne, «The robotized workstation MASTER for users with tetraplegia: Description and evaluation ", Journal of Rehabilitation Research and Development Vol. 36 No. 3, July 1999

[3] Rapport technique LAAS N92452, Décembre 1992, $70 \mathrm{p}$.

[4] Laurent DELAHOCHE, « coopération multicapteurs appliquée à la localisation des robots mobiles », thèse 
de doctorat de l'Université de Picardie Jules Verne, juillet 1997.

[5] Arnaud CLERENTIN, Laurent DELAHOCHE, Eric BRASSART, Claude PEGARD «Omnidirectional sensors cooperation for multi-target tracking », IEEE Int. Conf. on Multisensor Fusion and Integration for Intelligent Systems (MFI 2001), Baden-Baden, August 20-22, 2001

[6] Cyril DROCOURT, Laurent DELAHOCHE, Claude PEGARD, Arnaud CLERENTIN - « Mobile Robot Localization Based on an Omnidirectional Stereoscopic Vision Perception System »- Proc. of the IEEE International Conference on Robotics and Automation (ICRA 99), May 1999, p. 1329 - 1334.

[7] Morvan, JS, JP Guichard and V Torossian (1997). "Technical aids for the physically handicapped: a psychological study of the master robot." Int J Rehabil Res 20(2): 193-7.

[8] Rémy-Néris, O, GL Claire and B Busnel (1998). Evaluation APPROCHE des robots Master et Manus en France: résultats définitifs. Progrès en Médecine physique et de réadaptation. L. Simon, J. Pélissier and C. Hérisson. Paris, Masson: 465-469. 